\title{
Speaker Change Detection in Broadcast TV using Bidirectional Long Short-Term Memory Networks
}

\author{
Ruiqing Yin, Hervé Bredin, Claude Barras \\ LIMSI, CNRS, Univ. Paris-Sud, Université Paris-Saclay, F-91405 Orsay, France \\ firstname.lastnamedimsi.fr
}

\begin{abstract}
Speaker change detection is an important step in a speaker diarization system. It aims at finding speaker change points in the audio stream. In this paper, it is treated as a sequence labeling task and addressed by Bidirectional long short term memory networks (Bi-LSTM). The system is trained and evaluated on the Broadcast TV subset from ETAPE database. The result shows that the proposed model brings good improvement over conventional methods based on BIC and Gaussian Divergence. For instance, in comparison to Gaussian divergence, it produces speech turns that are $19.5 \%$ longer on average, with the same level of purity.
\end{abstract}

Index Terms: speaker diarization, speaker change detection, sequence labeling, recurrent neural network, LSTM

\section{Introduction}

Speaker diarization is the task of determining "who spoke when" in an audio stream that usually contains an unknown amount of speech from an unknown number of speakers $[1,2]$. Speaker change detection is an important part of speaker diarization systems. It aims at finding the boundaries between speech turns of two different speakers.

In recent years, the performance of state-of-the-art speech and speaker recognition systems has improved enormously thanks to the neural network (especially deep learning) approaches. The proposed system in [3] has achieved human parity in conversational speech recognition. The key to this system's performance is the systematic use of convolutional and Long Short-Term Memory (LSTM) neural networks. In speech recognition and natural language processing, LSTM has been used successfully for sequence labeling [4], language modeling [5] and machine translation [6]. However, existing speaker diarization systems do not take full advantages of these new techniques. [7] proposes an artificial neural network architecture to learn a feature transformation from MFCCs that is optimized for speaker diarization. However, the proposed system does not improve the initial change detection step, and rely on conventional methods presented in Section 2.

Our main contribution is introduced in Section 3 where we address speaker change detection as a binary sequence labeling task using Bidirectional Long Short-Term Memory recurrent neural networks (Bi-LSTM). Experiments on the ETAPE dataset are summarized in Section 4 and results are discussed in Section 5.

\section{Related work}

In conventional speaker change detection methods, one will use two adjacent sliding windows on the audio data, compute a distance between them, then decide (usually by thresholding the distance) whether the two windows originate from the same speaker. Gaussian divergence [8] and Bayesian Information Criterion (BIC) [9] have been used extensively in the literature to compute such a distance: they have both advantages of leading to good segmentation results and not requiring any training step (other than for tuning the threshold).

There were some recent attempts at improving over these strong baselines with supervised approaches. Desplanques et al. [10] investigate factor analysis and i-vector for speaker segmentation. We recently proposed in [11] to train a recurrent neural network called TristouNet to project any speech segment into a large-dimensional space where speech turns from the same (resp. different) speaker are close to (resp. far from) each other according to the euclidean distance. Replacing BIC or Gaussian divergence by the euclidean distance between TristouNet embeddings brings significant speaker change detection improvement. However, because they rely on relatively long adjacent sliding windows ( 2 seconds or more), all these methods tend to miss boundaries in fast speaker interactions.

Instead, we propose to address speaker change detection as a sequence labeling task, using LSTMs in the same way they have been successfully used for speech activity detection. In particular, our proposed approach is the direct translation of the work by Gelly et al. where they applied Bi-LSTMs on overlapping audio sequences to predict whether each frame corresponds to a speech region or a non-speech one [12].

\section{Speaker change detection as a sequence labeling problem}

Given an audio recording, speaker change detection aims at finding the boundaries between speech turns of different speakers. In Figure 1, the expected output of such a system would be the list of timestamps between spk1 \& spk2, spk2 \& spk1, and spk1 \& spk4.

\subsection{Sequence labeling}

Let $\mathbf{x} \in \mathcal{X}$ be the sequence of features extracted from the audio recording: $\mathbf{x}=\left(x_{1}, x_{2} \ldots x_{T}\right)$ where $T$ is the total number of features extracted from the sequence. Typically, $\mathbf{x}$ would be a sequence of MFCC features extracted on a short (a few milliseconds) overlapping sliding window ( $a k a$. frame). The speaker change detection task is then turned into a binary sequence labeling task by defining $\mathbf{y}=\left(y_{1}, y_{2} \ldots y_{T}\right) \in\{0,1\}^{T}$ such that $y_{i}=1$ if there is a speaker change during the $i$ th frame, and $y_{i}=0$ otherwise (see part B of Figure 1). The objective is then to find a function $f: \mathcal{X} \rightarrow \mathcal{Y}$ that matches a feature sequence to a label sequence. We propose to model this function $f$ as a recurrent neural network trained using the binary cross-entropy loss function:

$$
L=-\frac{1}{T} \sum_{i=1}^{T} y_{i} \log \left(f(\mathbf{x})_{i}\right)+\left(1-y_{i}\right) \log \left(1-f(\mathbf{x})_{i}\right)
$$


(A)

\begin{tabular}{|l|l|l|l|}
\hline spk1 & spk2 & spk1 & spk4 \\
\hline
\end{tabular}

(B)

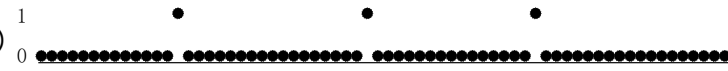

(C)

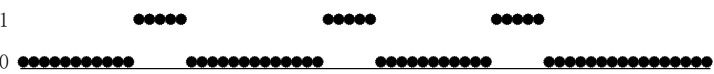

(D)

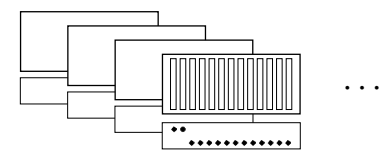

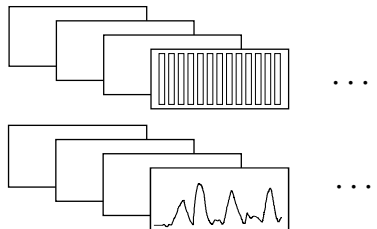

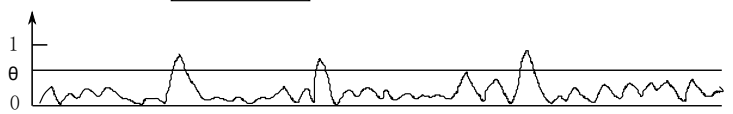

\begin{tabular}{|l|l|l|l|}
\hline seg1 & seg2 & seg3 & seg4 \\
\hline spk1 & spk2 & spk1 & spk4 \\
\hline
\end{tabular}

Figure 1: Training process (left) and prediction process (right) for speaker change detection. Details can be found in Section 3.

\subsection{Network architecture}

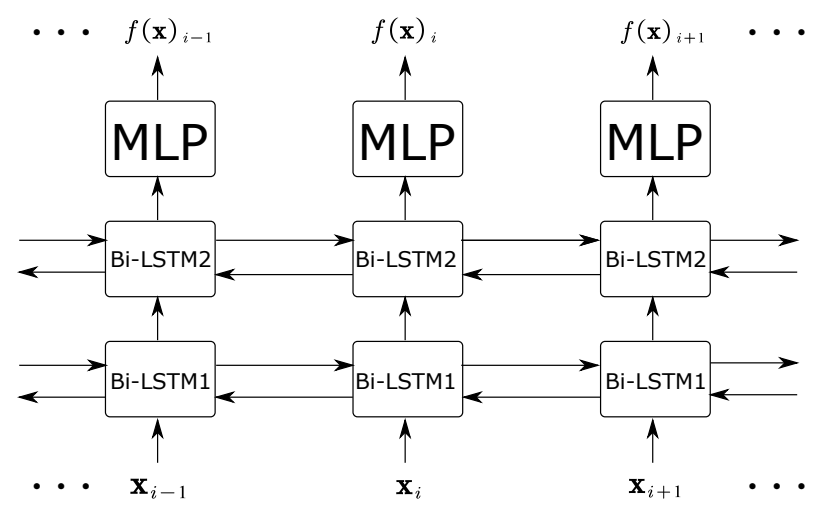

Figure 2: Model architecture

The actual architecture of the network $f$ is depicted in Figure 2. It is composed of two Bi-LSTM (Bi-LSTM 1 and 2) and a multi-layer perceptron (MLP) whose weights are shared across the sequence. Bi-LSTMs [13] allow to process sequences in forward and backward directions, making use of both past and future contexts. The output of both forward and backward LSTMs are concatenated and fed forward to the next layer. The shared MLP is made of three fully connected feedforward layers, using tanh activation function for the first two layers, and a sigmoid activation function for the last layer, in order to output a score between 0 and 1 .

\subsection{Class imbalance}

Since there are relatively few change points in the audio files, very little frames are in fact labeled as positive (1). For instance, in the training of the ETAPE dataset used in the experimental section, this represents only $0.4 \%$ of all frames. This class imbalance issue could be problematic when training the neural network. Moreover, one cannot assume that human annotation are precise at the frame level. It is likely that the actual location of speech turn boundaries is a few frames away from the one selected by the human annotators. This observation led most speaker diarization evaluation benchmarks $[14,15,16]$ to remove from evaluation a short collar (up to half a second) around each manually annotated boundary. Therefore, as de- picted in part $\mathrm{C}$ of Figure 1, the number of positive labels is increased artificially by labeling as positive every frame in the direct neighborhood of the manually annotated change point. We will further evaluate the impact of the size of this neighborhood in Section 5.

\subsection{Subsequences}

One well-publicized property of LSTMs is that they are able to avoid the vanishing gradients problem encountered by traditional recurrent neural networks $[17,4]$. Therefore, the initial idea was to train them on whole audio sequences at once but we found out that this has several limitations, including the limited number of training sequences, and the computational cost and complexity of processing such long sequences with variable lengths. Consequently, as depicted in part D of Figure 1, the long audio sequences are split into short fixed-length overlapping sequences. This has the additional benefit of increasing the variability and number of sequences seen during training, as is usually done with data augmentation for computer vision tasks. We will further discuss the advantages of this approach in Section 5.

\subsection{Prediction}

Once the network is trained, it can be used to perform speaker change detection as depicted in the right part of Figure 1. First, similarly to what is done during training, test files are split into overlapping feature sequences (part E of Figure 1). The network processes each subsequence to give a sequence of scores between 0 and 1 (part F of Figure 1). Because input sequences are overlapping, each frame can have multiple candidate scores; they are averaged to obtain the final frame-level score. Finally, local score maxima exceeding a pre-determined threshold $\theta$ are marked as speaker change points, as shown in part G of Figure 1. Parts $\mathrm{H}$ and I respectively represent the hypothesized and groundtruth speaker change points.

\section{Experiments}

\subsection{Dataset}

The ETAPE TV subset [18] contains 29 hours of TV broadcast (18h for training, 5.5h for development and 5.5h for test) from three French TV channels with news, debates, and entertainment. Fine "who speaks when" annotations were provided on a 
subset of the training and development set using the following two-steps process: automatic forced alignment of the manual speech transcription followed by manual boundaries adjustment by trained phoneticians. Overall, this leads to a training set of 13 h50 containing $\mathrm{N}=184$ different speakers, and a development set of $4 \mathrm{~h} 10$ containing 61 speakers (out of which 18 are also in the training set). Due to a coarser segmentation, the official test set is not used in this paper and the reported results are computed on the development set.

\subsection{Implementation details}

Feature extraction. 35-dimensional acoustic features are extracted every $16 \mathrm{~ms}$ on a $32 \mathrm{~ms}$ window using Yaafe toolkit [19]: 11 Mel-Frequency Cepstral Coefficients (MFCC), their first and second derivatives, and the first and second derivatives of the energy.

Network architecture. The model stacks two Bi-LSTMs and a multi-layer perceptron. Bi-LSTM1 has 64 outputs (32 forward and 32 backward). Bi-LSTM2 has 40 (20 each). The fully connected layers are 40-, 10- and 1-dimensional respectively.

Training. As introduced in Section 3, and unless otherwise stated, a positive neighborhood of $100 \mathrm{~ms}$ (50ms on both sides) is used around each change point, to partially solve the class imbalance problem. Subsequences for training are 3.2s long with a step of $800 \mathrm{~ms}$ (i.e. two adjacent sequences overlap by $75 \%$ ). The actual training is implemented in Python using the Keras toolkit [20], and we use the SMORMS3 [21] optimizer.

Baseline. Both BIC [9] and Gaussian divergence [8] baselines rely on the same set of features (without derivatives, because it leads to better performance), using two $2 \mathrm{~s}$ adjacent windows. We also report the result obtained by the TristouNet approach, that used the very same experimental protocol [11].

\subsection{Evaluation metrics}

Conventional evaluation metrics for the speaker change detection task are recall and precision. A hypothesized change point is counted as correct if it is within the temporal neighborhood of a reference change point. Both values are very sensitive to the actual size of this temporal neighborhood ( $a k a$. tolerance) quickly reaching zero as the tolerance decreases. It also means that it is very sensitive to the actual temporal precision of human annotators.

Following what was done in [11], we propose to use purity and coverage evaluation metrics (as defined in pyannote.metrics [22]) because they do not depend on a tolerance parameter and are more relevant in the perspective of a speaker diarization application. Purity [23] and coverage [24] were introduced to measure cluster quality but can also be adapted to the speaker change points detection task. Given $\mathcal{R}$ the set of reference speech turns, and $\mathcal{H}$ the set of hypothesized segments, coverage is defined as follows

$$
\operatorname{coverage}(\mathcal{R}, \mathcal{H})=\frac{\sum_{r \in \mathcal{R}} \max _{h \in \mathcal{H}}|r \cap h|}{\sum_{r \in \mathcal{R}}|r|}
$$

where $|s|$ is the duration of segment $s$ and $r \cap h$ is the intersection of segments $r$ and $h$. Purity is the dual metric where the role of $\mathcal{R}$ and $\mathcal{H}$ are interchanged. Over-segmentation (i.e. detecting too many speaker changes) would result in high purity but low coverage, while missing lots of speaker changes would decrease purity - which is critical for subsequent speech turn agglomerative clustering.

\section{Results}

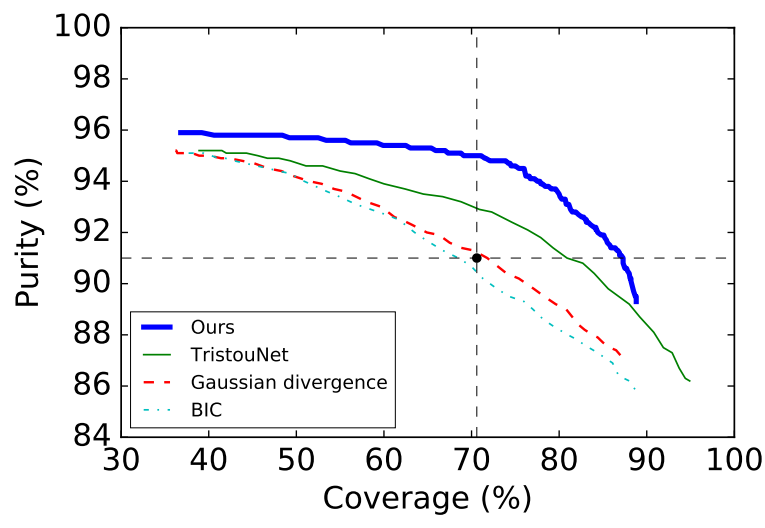

Figure 3: Speaker change detection on ETAPE development set

All tested approaches (including the one we propose) rely on a peak detection step (keeping only those whose value is higher than a given threshold $\theta$ ). Curves in Figure 3 were obtained by varying this very threshold $\theta$. Our proposed solution clearly outperforms BIC-, divergence-, and TristouNetbased approaches, whatever the operating point. Notice how it reaches a maximum purity of $95.8 \%$, while all others are stuck at $95.1 \%$. This is explained by the structural limitations of approaches based on the comparison of two adjacent windows: it is not possible for them to detect two changes if they belong to the same window. Our proposed approach is not affected by this issue.
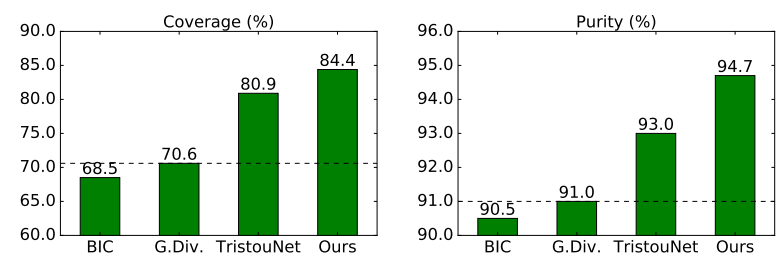

Figure 4: Left: coverage at $91.0 \%$ purity. Right: purity at $70.6 \%$ coverage.

Figure 4 summarizes the same set of experiments in a different way, showing purity at $70.6 \%$ coverage, and coverage at $91.0 \%$ purity. Those two values are marked by the horizontal and vertical lines in Figure 3 and were selected because they correspond to the operating point of the divergence-based segmentation module of our in-house multi-stage speaker diarization system [25]. Our approach improves both purity and coverage. For instance, in comparison to Gaussian divergence, it produces speech turns that are $19.5 \%$ longer on average, with the same level of purity.

We did try to integrate our contribution into our in-house speaker diarization system. Unfortunately, the overall impact on the complete system in terms of diarization error rate is very limited. This may be because the subsequent clustering module was optimized jointly with the divergence-based segmentation step, expecting a normal distribution of features in each segment 
- which has no reason to be true for the ones obtained through the use of LSTMs.

\subsection{Fixing class imbalance}

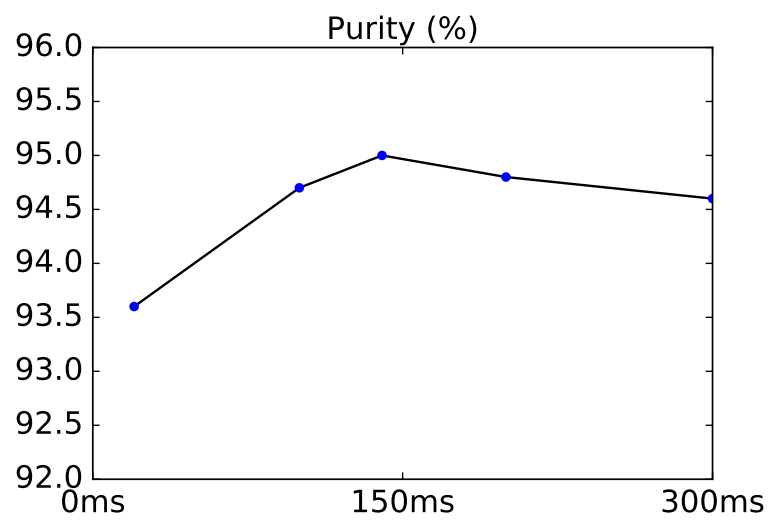

Figure 5: Purity at $70.6 \%$ coverage for different balancing neighborhood size

As discussed in Section 3, to deal with the class imbalance problem, we artificially increased the number of positive labels during training by labeling as positive every frame in the direct neighborhood of each change point. Figure 5 illustrates the influence of the duration of this neighborhood on the segmentation purity, given that coverage is fixed at $70.6 \%$. It shows a maximum value for a neighborhood of around $140 \mathrm{~ms}$. One should also notice that, even without any class balancing effort, the proposed approach is still able to reach $93.6 \%$ purity, outperforming the other three tested approaches: the class imbalance issue is not as problematic as we initially expected.

\section{2. "The Unreasonable Effectiveness of LSTMs"}

As Karpathy would put it ${ }^{1}$, the proposed approach seems unreasonably effective. Even though LSTMs do rely on an internal memory, it is still surprising that they perform that well for speaker change detection, given that, at a particular time step $i$, all they see is the current feature vector $\mathbf{x}_{i}$. We first thought that concatenating features from adjacent frames would be beneficial, but this did not bring any significant improvement. The internal memory mechanism is powerful enough to collect and keep track of contextual information.

This is further highlighted in Figure 6 that plots the expected absolute difference between predicted scores $f(\mathbf{x})_{i} \in$ $[0,1]$ and reference labels $\mathbf{y}_{i} \in\{0,1\}$, as a function of the position $i$ in the sequence: $\delta(i)=\mathbb{E}_{\mathbf{x}, \mathbf{y}}\left(\left|f(\mathbf{x})_{i}-\mathbf{y}_{i}\right|\right)$. It clearly shows that the proposed approach performs better in the middle than at the beginning or the end of the sequence, quickly reaching a plateau as enough contextual information has been collected. This anticipated behavior justifies after the fact the use of strongly overlapping subsequences - making sure that each time step falls within the best performing region at least once.

\footnotetext{
$1_{\text {karpathy.github.io/2015/05/21/rnn-effectiveness }}$
}

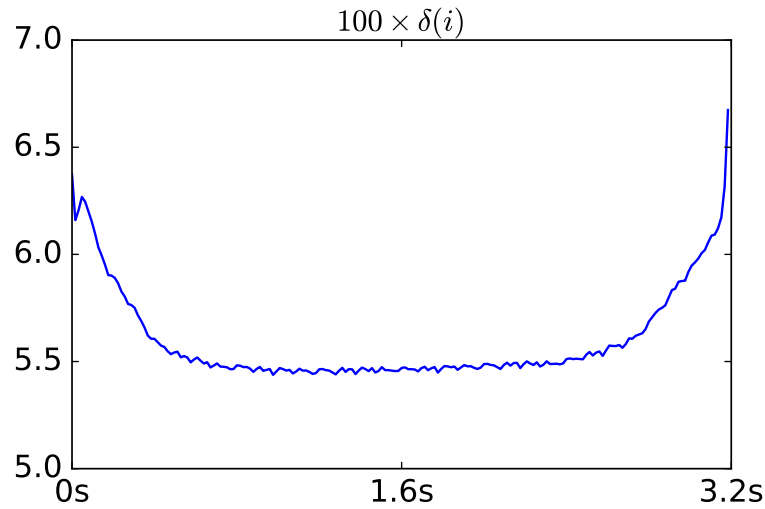

Figure 6: Expected absolute difference between prediction score and reference label, as a function of the position in the $3.2 \mathrm{~s}$ subsequence.

\section{Conclusion and future work}

We have developed a speaker change detection approach using bidirectional long short-term memory networks. Experimental results on the ETAPE dataset led to significant improvements over conventional methods (e.g., based on Gaussian divergence) and recent state-of-the-art results based on TristouNet embeddings ( [11] also using LSTMs).

While neural networks are often considered as "magic" black boxes, we tried in Section 5.2 to better understand why these approaches are so powerful, despite their apparent simplicity. Yet, a lot remains to be done to really fully understand how the internal memory cells are actually used to gather and use the contextual information needed for detecting speaker changes.

Finally, despite major improvements of the speaker change detection module, its impact on the overall speaker diarization system is minor, possibly because segments obtained by LSTMs are not adapted to standard BIC- or CLR-based speaker diarization approach. We plan to investigate LSTM-based speech turn embeddings like TristouNet to fully benefit from this improved segmentation.

\section{Reproducible research}

Code to reproduce the results of this paper is available here: github.com/yinruiqing/change_detection

\section{Acknowledgements}

This work was partly supported by ANR through the ODESSA (ANR-15-CE39-0010) and MetaDaTV (ANR-14-CE24-0024) projects. We thank M. Gregory Gelly for sharing useful ideas and experience on Bi-LSTM networks applied to speech processing. 


\section{References}

[1] S. E. Tranter and D. A. Reynolds, "An overview of automatic speaker diarization systems," IEEE Transactions on audio, speech, and language processing, vol. 14, no. 5, pp. 1557-1565, 2006.

[2] X. Anguera, S. Bozonnet, N. Evans, C. Fredouille, G. Friedland, and O. Vinyals, "Speaker diarization: A review of recent research," IEEE Transactions on Audio, Speech, and Language Processing, vol. 20, no. 2, pp. 356-370, 2012.

[3] W. Xiong, J. Droppo, X. Huang, F. Seide, M. Seltzer, A. Stolcke, D. Yu, and G. Zweig, "Achieving Human Parity in Conversational Speech Recognition,” Tech. Rep., February 2017. [Online]. Available: https://www.microsoft.com/en-us/research/publication/ achieving-human-parity-conversational-speech-recognition-2/

[4] A. Graves, "Neural Networks," in Supervised Sequence Labelling with Recurrent Neural Networks. Springer, 2012, pp. 15-35.

[5] M. Sundermeyer, R. Schlüter, and H. Ney, "LSTM Neural Networks for Language Modeling." in Interspeech 2012, 13th Annual Conference of the International Speech Communication Association, 2012, pp. 194-197.

[6] I. Sutskever, O. Vinyals, and Q. V. Le, "Sequence to sequence learning with neural networks," in Advances in neural information processing systems, 2014, pp. 3104-3112.

[7] S. H. Yella, A. Stolcke, and M. Slaney, "Artificial neural network features for speaker diarization," in Spoken Language Technology Workshop (SLT), 2014 IEEE. IEEE, 2014, pp. 402-406.

[8] M. A. Siegler, U. Jain, B. Raj, and R. M. Stern, "Automatic segmentation, classification and clustering of broadcast news audio," in Proc. DARPA speech recognition workshop, vol. 1997, 1997.

[9] S. Chen and P. Gopalakrishnan, "Speaker, environment and channel change detection and clustering via the bayesian information criterion," in Proc. DARPA Broadcast News Transcription and Understanding Workshop, vol. 8. Virginia, USA, 1998, pp. 127132.

[10] B. Desplanques, K. Demuynck, and J.-P. Martens, "Factor analysis for speaker segmentation and improved speaker diarization," in Interspeech 2015, 16th Annual Conference of the International Speech Communication Association, 2015, pp. 3081-3085.

[11] H. Bredin, "TristouNet: Triplet Loss for Speaker Turn Embedding," in ICASSP 2017, IEEE International Conference on Acoustics, Speech, and Signal Processing, New Orleans, USA, March 2017.

[12] G. Gelly and J.-L. Gauvain, "Minimum word error training of RNN-based voice activity detection." in Interspeech 2015, 16th Annual Conference of the International Speech Communication Association, 2015, pp. 2650-2654.

[13] A. Graves, N. Jaitly, and A.-r. Mohamed, "Hybrid speech recognition with deep bidirectional LSTM," in Automatic Speech Recognition and Understanding (ASRU), 2013 IEEE Workshop on. IEEE, 2013, pp. 273-278.
[14] J. G. Fiscus, N. Radde, J. S. Garofolo, A. Le, J. Ajot, and C. Laprun, "The Rich Transcription 2005 spring meeting recognition evaluation," in International Workshop on Machine Learning for Multimodal Interaction (MLMI). Springer, 2005, pp. 369389.

[15] O. Galibert, J. Leixa, G. Adda, K. Choukri, and G. Gravier, "The ETAPE speech processing evaluation." in LREC, 2014, pp. 3995 3999.

[16] O. Galibert, "Methodologies for the evaluation of speaker diarization and automatic speech recognition in the presence of overlapping speech." in Interspeech 2013, 14th Annual Conference of the International Speech Communication Association, 2013, pp. 1131-1134.

[17] S. Hochreiter and J. Schmidhuber, "Long short-term memory," Neural computation, vol. 9, no. 8, pp. 1735-1780, 1997.

[18] G. Gravier, G. Adda, N. Paulson, M. Carré, A. Giraudel, and O. Galibert, "The ETAPE corpus for the evaluation of speech-based TV content processing in the French language," in LREC - Eighth international conference on Language Resources and Evaluation, Turkey, 2012, p. na. [Online]. Available: https://hal.archives-ouvertes.fr/hal-00712591

[19] B. Mathieu, S. Essid, T. Fillon, J. Prado, and G. Richard, "YAAFE, an Easy to Use and Efficient Audio Feature Extraction Software." in ISMIR 2010, 11th International Society for Music Information Retrieval Conference, 2010, pp. 441-446.

[20] F. Chollet, "Keras," 2015. [Online]. Available: https://github. com/fchollet/keras

[21] S. Funk, "Rmsprop loses to SMORMS3," 2015. [Online]. Available: http://sifter.org/ simon/journal/20150420.html

[22] H. Bredin, "pyannote.metrics: a toolkit for reproducible evaluation, diagnostic, and error analysis of speaker diarization systems," in Interspeech 2017, 18th Annual Conference of the International Speech Communication Association, Stockholm, Sweden, August 2017. [Online]. Available: http://pyannote. github.io/pyannote-metrics

[23] M. Cettolo, "Segmentation, classification and clustering of an Italian broadcast news corpus," in Content-Based Multimedia Information Access-Volume 1, 2000, pp. 372-381.

[24] J.-L. Gauvain, L. Lamel, and G. Adda, "Partitioning and transcription of broadcast news data." in ICSLP 1998, 5th International Conference on Spoken Language Processing, vol. 98, no. 5, 1998, pp. $1335-1338$.

[25] C. Barras, X. Zhu, S. Meignier, and J. L. Gauvain, "Multi-Stage Speaker Diarization of Broadcast New," IEEE Transactions on Audio, Speech and Language Processing, vol. 14, no. 5, pp. 1505 1512, Sep. 2006. 